\title{
Investigating the spatial variability of the time-scaling properties in Italian seismicity
}

\author{
L. Telesca and V. Lapenna \\ Institute of Methodologies for Environmental Analysis, National Research Council, Tito Scalo (PZ), Italy \\ Received: 9 August 2004 - Revised: 13 October 2004 - Accepted: 11 November 2004 - Published: 16 November 2004 \\ Part of Special Issue "Seismicity pattern dynamics"
}

\begin{abstract}
Significant power-law long-range correlated structures have been identified in the Italian seismicity from 1983 to 2003. We performed the Allan Factor Analysis and the Detrended Fluctuation Analysis on both the full and the aftershock-depleted seismic data, extracted by the INGV (National Institute of Geophysics and Volcanology) instrumental catalog.

Our findings suggest that (i) the time-scaling behaviour characterizes mainly the Apennine chain; (ii) the timecorrelated behaviour of the seismicity is persistent, and this implies that the dynamics underlying the seismic phenomenology is characterized by the presence of "positive feedback mechanisms"; (iii) significant time correlation is not simply related with the mainshock/aftershock mechanisms.
\end{abstract}

\section{Introduction}

Several features account for the complexity in earthquake sequences. The Gutenberg-Richter law (Gutenberg and Richter, 1944) states that the distribution of the magnitudes of earthquakes follows a power-law. The epicenter distribution is fractal in space and also the faults have a fractal-like structure (Kagan and Jackson, 1991). The Omori's law formalizes the presence of short-range temporal correlation between earthquakes, since after a main large shock a series of aftershocks occurs, with a power-law time-frequency decay (Utsu et al., 1995).

Therefore, tectonic processes are considered to display fractal properties in time (e.g. Godano and Caruso, 1995; Öncel et al., 1996; Wilson and Dominic, 1998). Identifying whether the time-occurrences of earthquakes are independent on each other or not, leads to the assessment of the statistical distribution ruling the event occurrence. Several distributions have been used to model seismic activity. Poisson statistics

Correspondence to: L. Telesca

(ltelesca@imaa.cnr.it) has been undoubtly the most extensively used, since, in many cases, for large events a simple discrete Poisson distribution provides a close fit (Boschi et al., 1995). But the complexity of the earthquake dynamics has revealed the presence of time-clustering at both short and long time-scales (Kagan and Jackson, 1991). The analysis of the temporal variations of the scaling properties of earthquakes has been used to characterize the main features of seismicity and to bring us insight the inner dynamics of seismotectonic activity. The evolution of scaling exponents with respect to time has revealed the increase of the time-clustering feature of seismicity corresponding to large events, mostly due to the aftershock activation (Telesca et al., 2001a). The depth-dependent variation of the time-clustering behaviour of seismicity has been interpreted in relation with the brittle and ductile behaviour of the crust versus depth (Telesca et al., 2001b).

The objective of this study is to analyze the spatial distribution of the long-range time scaling properties of the Italian seismicity, investigated using the Allan Factor Analysis (AFA) and the Detrended Fluctuation Analysis (DFA). Both the methods are suited to analyze observational time series, which are often affected by nonstationarities.

\section{Methods}

The standard technique to investigate the temporal properties of a time series is the power spectral density (PSD) S(f), obtained by means of a Fourier transform of the signal. The PSD informs on how the power is concentrated at various frequency bands. This information allows one to identify periodic, multi-periodic or non-periodic behaviours. Usually the logarithmic power spectrum plot is used to better distinguish between the broadband and periodic components. The power-law dependence (linear on a log-log plot) of the PSD, given by $S(f) \sim f^{-\alpha}$, is a hallmark of the presence of timescaling properties in the data. The properties of the signal can be further classified in terms of the numerical value of the spectral exponent $\alpha$ : if $\alpha=0$, the signal is a realization of 
a white noise process, and is not characterized by any kind of time correlation; if $\alpha>0$, the signal possesses the tendency for repeating the sign of its fluctuations (i.e. if it increases in one period it will very likely increase in the next period), and, thus, it is persitstent; if $\alpha<0$, the signal is antipersistent and the fluctuations of opposite signs tend to alternate (Havlin et al., 1999). Therefore, the spectral exponent $\alpha$ is a quantitative parameter, which informs on the type of the scaling behaviour intrinsic in the data.

The crucial question is: how to estimate the spectral exponent $\alpha$ for a seismic sequence, which is a point process and the simple application of the Fourier Transform is not possible?

The question can be equivalent to the following: what parameter can be used to significantly describe the scaling properties of a seismic sequence?

The answer to this question is: it depends on the type of representation of the seismic sequence.

\subsection{Point process representation and Allan Factor Analysis}

A temporal point process describes events that occur at some random locations in time (Cox and Isham, 1980), and is expressed by a finite sum of Dirac's delta functions centered on the occurrence times $t_{i}$, with amplitude $\mathrm{A}_{i}$ proportional to the magnitude of the earthquake:

$y(t)=\sum_{i=1}^{N} A_{i} \delta\left(t-t_{i}\right)$,

where $\mathrm{N}$ represents the number of events recorded. In this representation we assume that the seismic events are the events of interest and there is an objective "clock" for the timing of each event.

In the framework of this representation, the Allan Factor Analysis (AFA) can be used. Let us divide the time axis into equally spaced contiguous counting windows of duration $\tau$, and produce a sequence of counts $\left\{\mathrm{Z}_{k}(\tau)\right\}$, with $\mathrm{Z}_{k}(\tau)$ denoting the number of earthquakes in the $\mathrm{k}$-th window:

$Z_{k}(\tau ; t)=\int_{t_{k-1}}^{t_{k}} \sum_{j=1}^{n} \delta\left(t-t_{j}\right) d t$.

The Allan Factor (AF) is a measure which can be used to distinguish fractal from Poissonian temporal fluctuations in point processes. This factor is defined as the variance of successive counts for a specified counting time $\tau$ divided by twice the mean number of events in that counting time:

$A F(\tau)=\frac{<\left(Z_{k+1}(\tau)-Z_{k}(\tau)\right)^{2}>}{2<Z_{k}(\tau)>}$.

The AF of a fractal point process varies with the counting time $\tau$ with a power-law form:

$A F(\tau)=1+\left(\frac{\tau}{\tau_{1}}\right)^{\alpha}$.
The monotonic power-law increase is representative of the presence of fluctuations on many time scales (Lowen and Teich, 1995); $\tau_{1}$ is the so-called fractal onset time, and marks the lower limit for significant scaling behaviour in the AF, so that for $\tau \ll \tau_{1}$ the clustering property becomes negligible within these timescales (Thurner et al., 1997). For Poissonian processes the $\mathrm{AF}$ assumes approximately values near or below unity for all counting times $\tau$. From Eq. (4), the calculation of $\alpha$ can be performed by estimating the slope of the straight line that fits in a least square sense the AF curve, plotted in log-log scales. Of course, only the linear part of the curve will be considered to calculate the fractal exponent.

\subsection{Interevent interval representation and detrended fluctu-} ation analysis

This approach considers the interspike intervals (the times between successive events) and uses the event number as an index of the time. With this approach the method of the Detrended Fluctuation Analysis (DFA) can be used.

The DFA performs the clustering analysis on the series of the interevent intervals. It was proposed by Peng et al. (1995), and avoids spurious detection of correlations that are artifacts of nonstationarity, that often affects experimental data. Such trends have to be well distinguished from the intrinsic fluctuations of the system in order to find the correct scaling behaviour of the fluctuations. Very often we do not know the reasons for underlying trends in collected data and we do not know the scales of underlying trends. DFA is a method for determining the scaling behaviour of data in the presence of possible trends without knowing their origin and shape.

The methodology operates on the time series $x(i)$, where $\mathrm{i}=1,2, \ldots, \mathrm{N}$ and $\mathrm{N}$ is the length of the series. With $\mathrm{x}_{\text {ave }}$ we indicate the mean value

$x_{\mathrm{ave}}=\frac{1}{N} \sum_{k=1}^{N} x(k)$.

The signal is first integrated

$y(k)=\sum_{i=1}^{k}\left[x(i)-x_{\text {ave }}\right]$.

Next, the integrated time series is divided into boxes of equal length $\mathrm{n}$. In each box a least-squares line is fit to the data, representing the trend in that box. The y coordinate of the straight line segments is denoted by $\mathrm{y}_{n}(\mathrm{k})$. Next we detrend the integrated time series $\mathrm{y}(\mathrm{k})$ by subtracting the local trend $\mathrm{y}_{n}(\mathrm{k})$ in each box. The root-mean-square fluctuation of this integrated and detrended time series is calculated by

$F(n)=\sqrt{\frac{1}{N} \sum_{k=1}^{N}\left[y(k)-y_{n}(k)\right]^{2}}$.

Repeating this calculation over all box sizes, we obtain a relationship between $\mathrm{F}(\mathrm{n})$, that represents the average fluctuation 


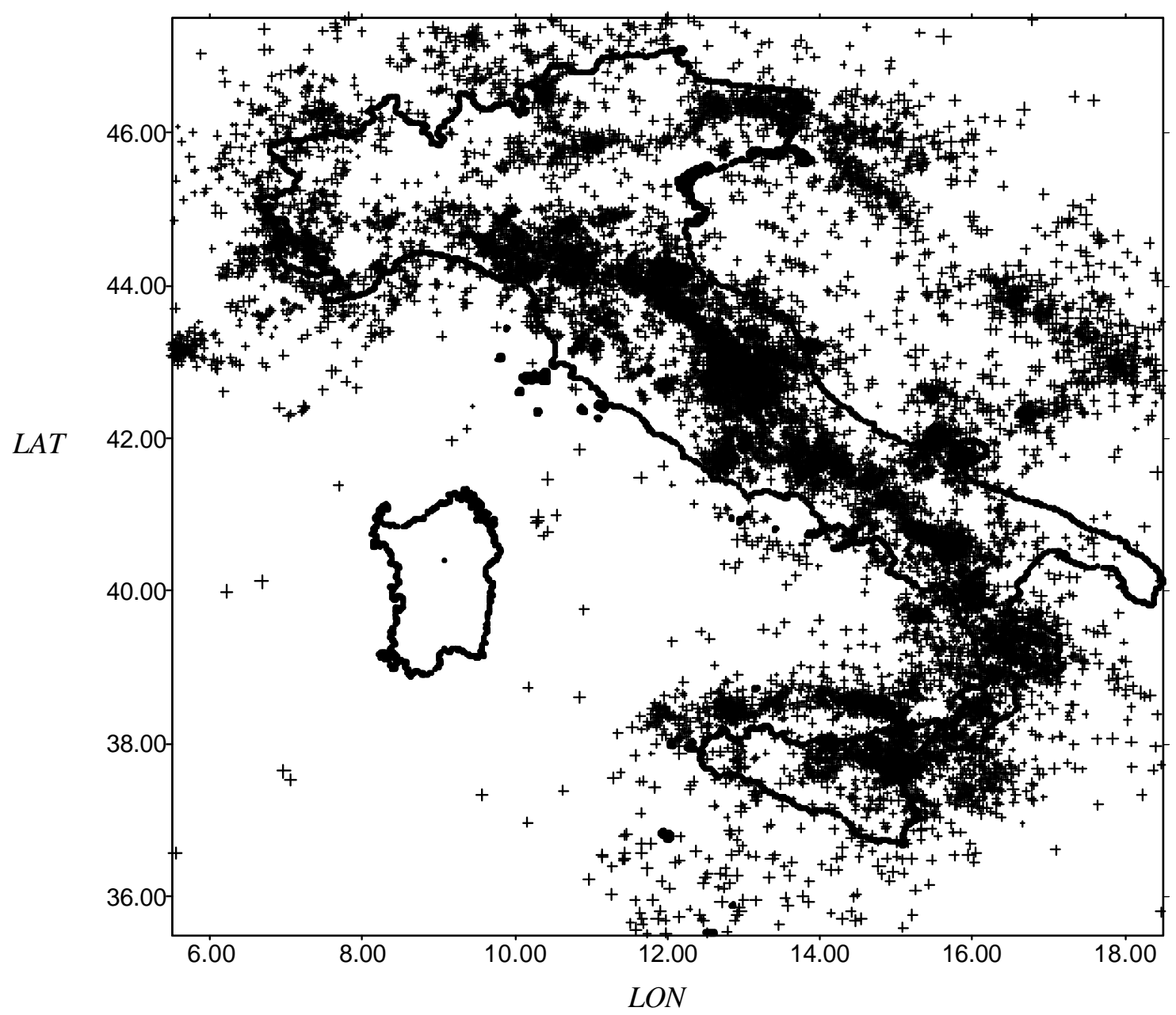

Fig. 1. Epicentral distribution of earthquakes $(M \geq 2.4)$ occurred in Italy over the period 1983-2003.

as a function of box size, and the box size $n$. If $F(n)$ behaves as a power-law function of $\mathrm{n}$, data present scaling:

$F(n) \propto n^{d}$.

Under these conditions the fluctuations can be described by the scaling exponent $\mathrm{d}$, representing the slope of the line fitting $\log F(n)$ to $\log n$. For a white noise process, $d=0.5$. If there are only short-range correlations, the initial slope may be different from 0.5 but will approach 0.5 for large window sizes. $d>0.5$ indicates the presence of persistent long-range correlations, meaning that a large (compared to the average) value is more likely to be followed by large value and vice versa. $\mathrm{d}<0.5$ indicates the presence of antipersistent longrange correlations, meaning that a large (compared to the average) value is more likely to be followed by small value and vice versa.

\section{Data analysis}

We have investigated the shallow (depth $\leq 100 \mathrm{~km}$ ) Italian seismicity from 1983 to 2003 , considering the earthquakes contained in a polygonal area surrounding the Italian coasts and borders within which the National Institute of Geophysics and Volcanology (INGV) has furnished reliable locations for the events of magnitude $\mathrm{M} \geq 2.4$, that represents the minimum magnitude for which the catalogue can be considered complete. The spatial distribution of the earthquakes, analysed in the present paper, is shown in Fig. 1. We studied the spatial variation of the time-scaling properties of the seismicity data of the full and aftershock-depleted catalogues. The analysis over the aftershock-depleted catalogue has been performed, because the aftershock clusters could bias the results, since the main shocks are followed by a large number of events (Breitenberg, 2000). A possible method to eliminate the aftershocks is to use a space-time rectangular or circular window, dependent on the magnitude of the mainshock (Gardner and Knopoff, 1976). This method has been improved by means of a dynamic aftershock clustering algorithm, which considers the peculiarity of each main shock concerning the extent of the aftershocks in space and time (Reasenberg, 1985). The method of Reasenberg (1985) is based on a physical basis, which considers each earthquake capable to generate an alteration of the surrounding stress 


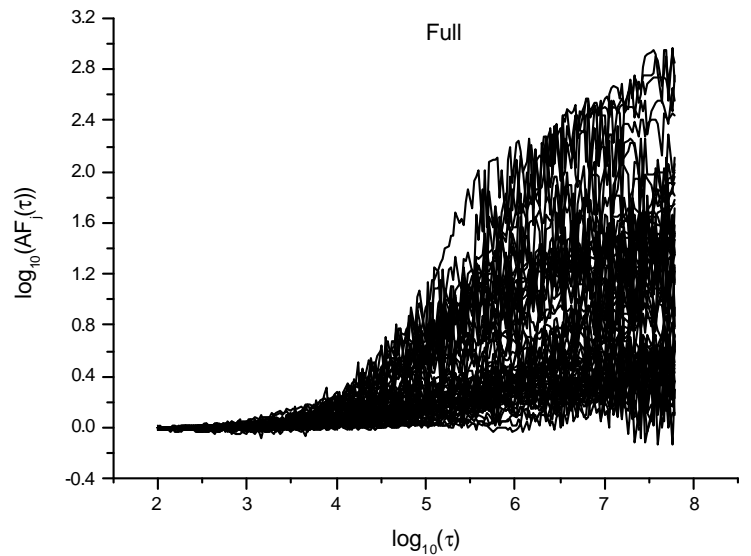

a)

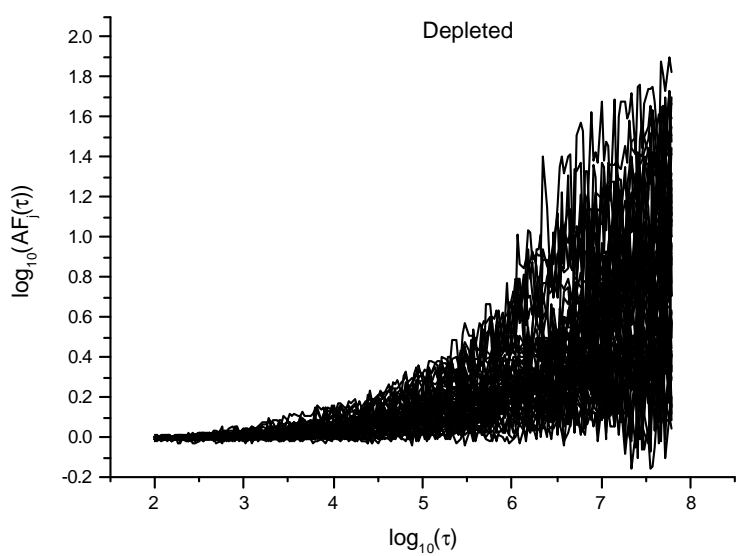

b)

Fig. 2. AF curves vs. counting time $\tau$ in the full (a) and the aftershock-depleted (b) Italian catalogues.

field that may trigger a further seismic event, which nucleates in its surroundings a modified stress field. The areal and time extent for which the event can trigger a following event is called interaction zone of the earthquake, whose length scale is proportional to the source dimension, and the temporal scale is determined with a probabilistic model based on Omori's law. Thus, we applied the Reasenberg's algorithm to remove aftershocks from the Italian catalogue. This method recognizes clusters in space-time in an earthquake catalog. It is intended for use in removing aftershocks or "declustering" the catalog. The method depends on a number of subjectively defined parameters. In our case we selected 10 days as the maximum look-ahead time for clustered events, 2.4 as the "effective" lower magnitude cutoff for the catalog and $10 \mathrm{~km}$ as the interaction radius of dependent events.

Since our study is aimed to analyze the spatial variation of the time-scaling properties of seismicity, we covered the Italian territory with a grid of cells having a size of $0.5^{\circ} \times 0.5^{\circ}$.

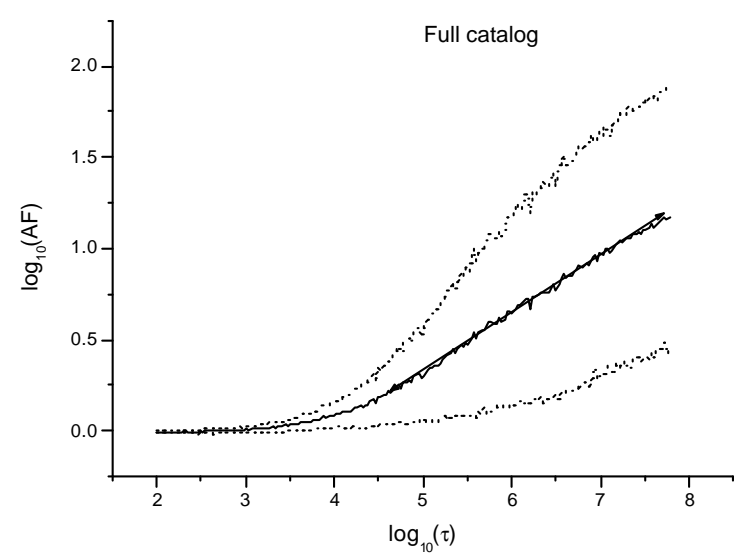

a)

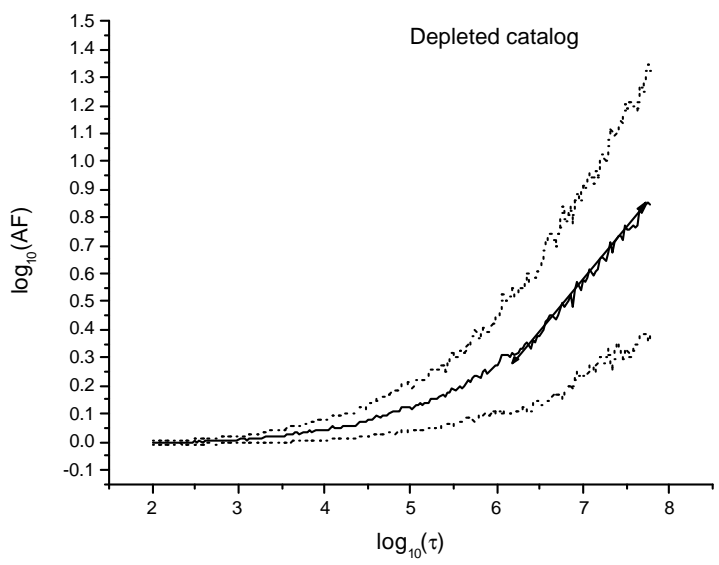

b)

Fig. 3. Mean AF curve $( \pm 1 \sigma)$ of the (a) full and (b) aftershockdepleted seismic catalogue.

We calculated the scaling exponents $\alpha$ and $\mathrm{d}$, as explained in the previous section, only if the cell contained a minimum of 100 events. A question naturally arises on how large are the statistical errors of the exponents for short series, where the statistics is expected to be poor. Varotsos et al. (2003) have analyzed the dependence of the estimate of the scaling exponents on the size, and they found that the statistical error depends on the size of the series only, assuming value of $0.16,0.10$ and 0.07 for lengths $\mathrm{N}=50,100$ and 200 respectively. Therefore, the choice of $\mathrm{N}=100$ as the minimum length of the series seems sufficient to get reliable estimates of the scaling exponents.

\subsection{Allan Factor Analysis}

In order to select the range of timescales involved in the scaling behaviour of the cells, we firstly calculated the AF curves for all the cells, satisfying the constraint on the minimum number of events. Figure 2 shows the AF curves for all the 

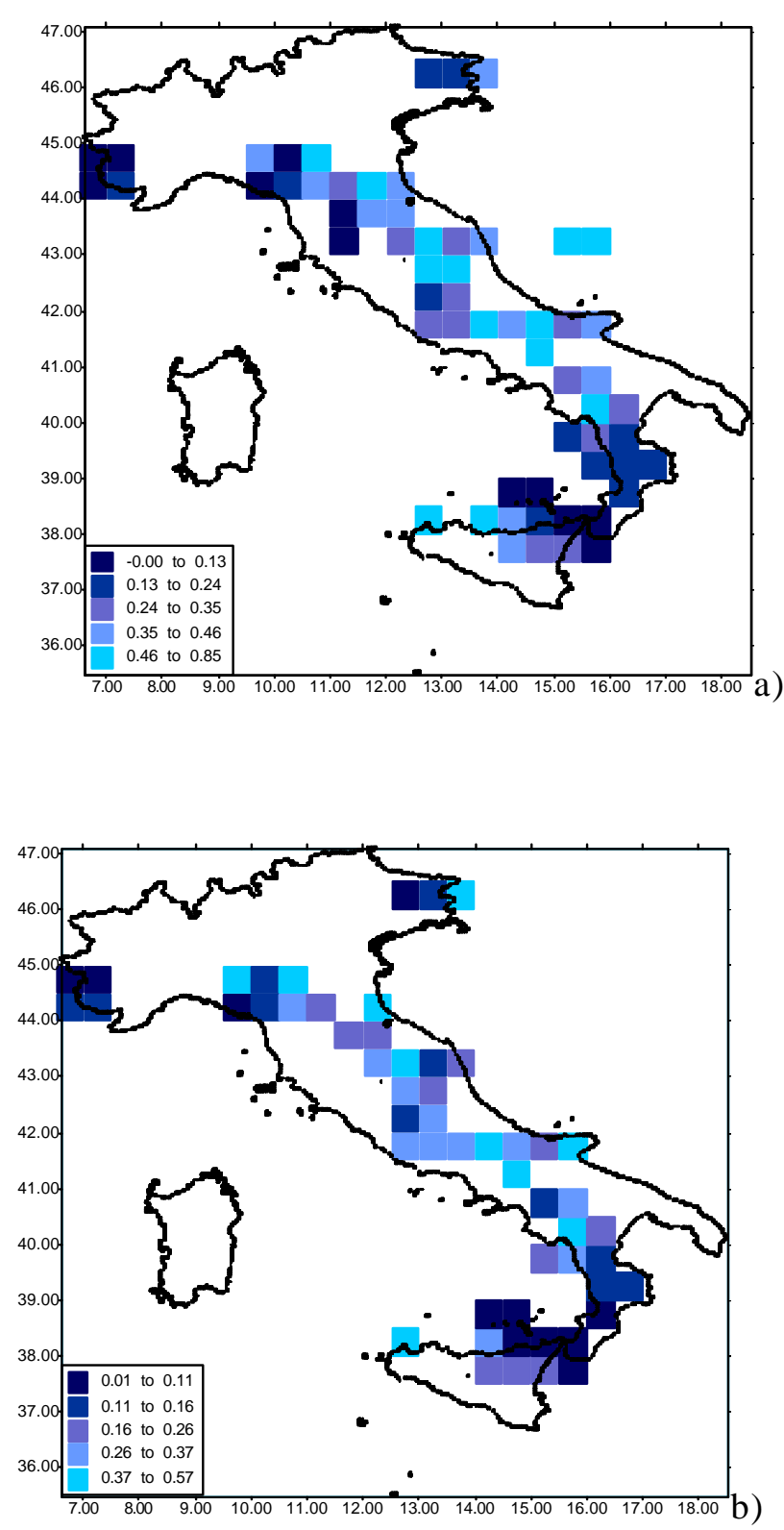

Fig. 4. Map of Italian territory showing the $\alpha$ variability: (a) full and (b) depleted seismic catalogues.

cells in the full (Fig. 2a) and aftershock-depleted (Fig. 2b) cases. Figure 3 shows the mean AF curve $( \pm 1 \sigma)$ for the full (a) and the depleted (b) case. We observe that the timescale range is long enough in both cases (about 4 decades in the full case and almost 2 decades in the depleted case), thus giving reliable estimates of the scaling exponents. The range in the full case is larger than that in the depleted case because the aftershocks introduce short-term correlations, and so produce an enlargement of the range of the correlation times. Therefore, we calculated for each cell the scaling exponent $\alpha$ for timescales starting from $10^{4.5} \mathrm{~s}$ and from $10^{6} \mathrm{~s}$ in the full and depleted case respectively. Figure 4 shows the spatial variation of the scaling exponent $\alpha$, estimated by

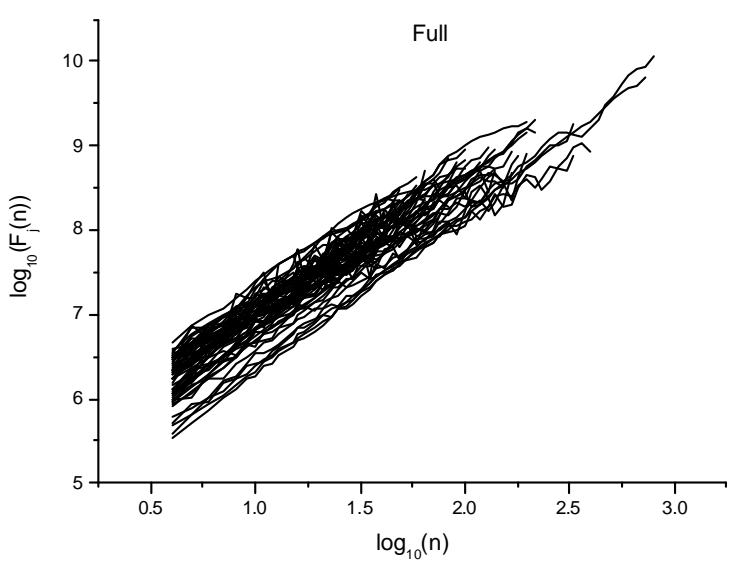

a)

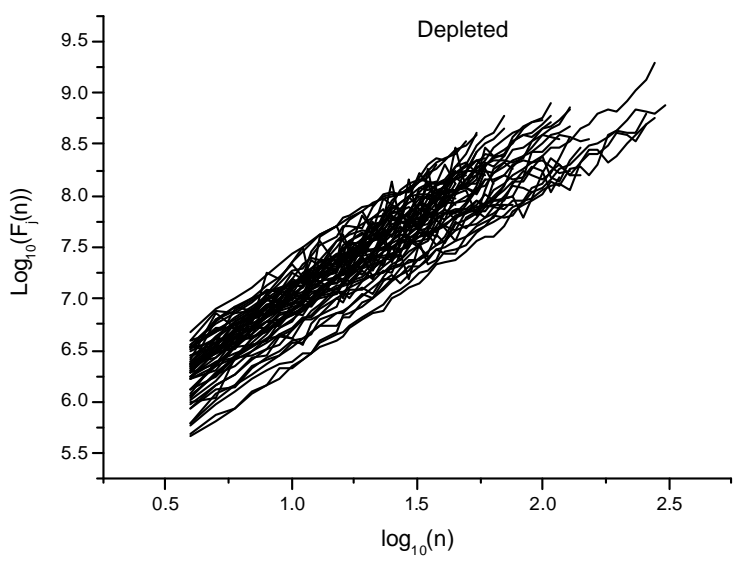

b)

Fig. 5. DFA curves in the (a) full and (b) afteshock-depleted seismic catalogues.

means of the AFA in the full case (a) and in the depleted case (b). Some cells are present in the full map, but not in the depleted one, since the aftershock depletion procedure has reduced their number of earthquakes. We observe a certain variability in the range between 0 and 1 of the scaling exponents from one cell to another, suggesting different types of time-correlation; in both cases the higher correlation degree is in central Italy, where recently a strong earthquake occurred in 1997. The maximum $\alpha$ values changes from 0.85 (full case) to 0.57 (depleted case), indicating that the deletion of the aftershocks causes the lowering of the strength of the time-correlation.

\subsection{Detrended Fluctuation Analysis}

As in the AFA, we firstly calculated the DFA curves for all the cells with a minimum number of 100 events, and plotted them to check the presence of scaling in all the cells and their global range. Figure 5 shows in the full (a) and depleted (b) case the fluctuation functions versus the index $n$. The scaling behaviour involves all the curves at all the scales. 

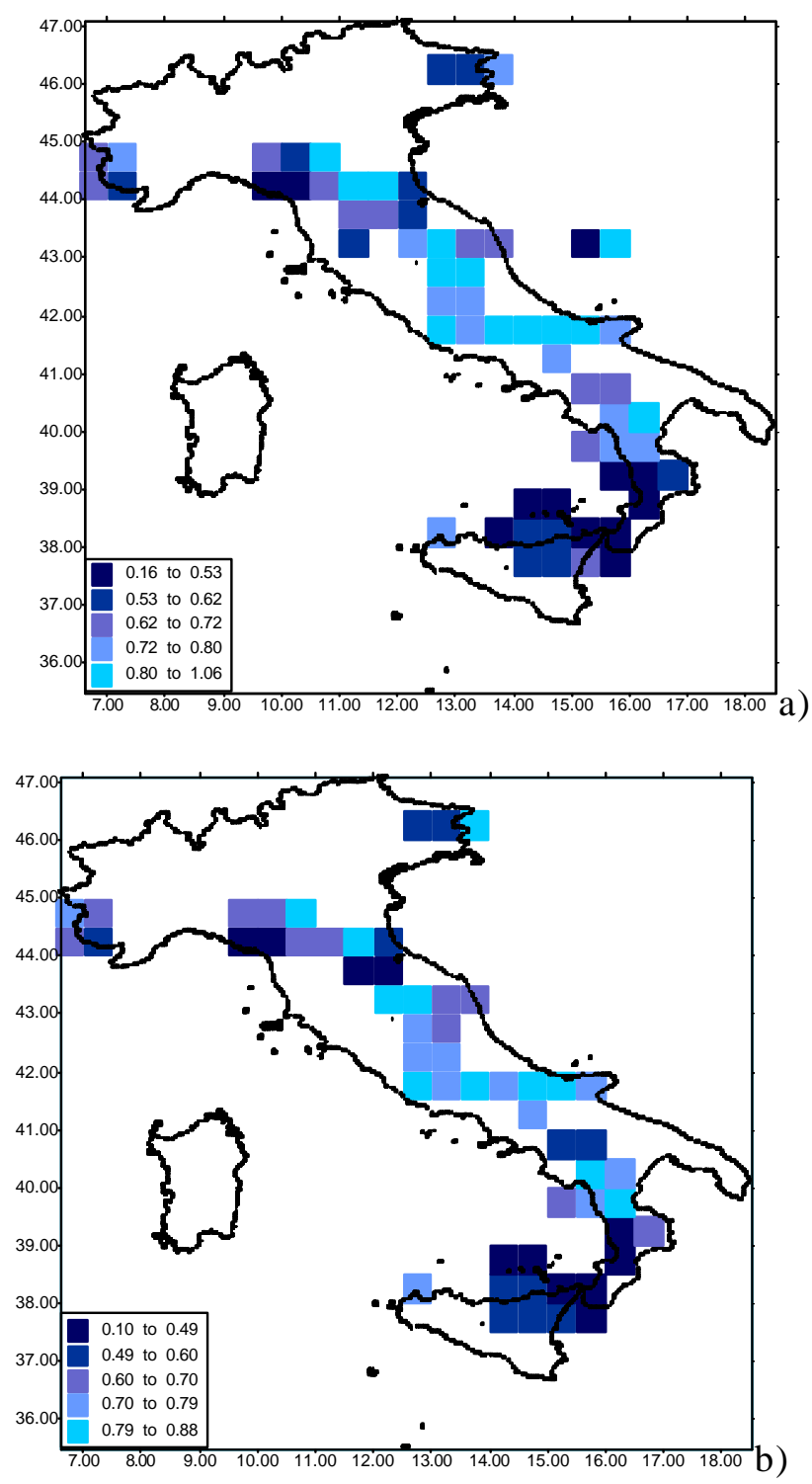

Fig. 6. Map of Italian territory showing the d variability: (a) full and (b) depleted seismic catalogues.

Figure 6 shows the spatial variation of the scaling exponent $\mathrm{d}$, estimated by means of the DFA in the full (a) and in the depleted case (b). The cells show a variability in the persistence properties. The maximum value of the $d$ exponent, indicating the degree of persistence, becomes smaller in the depleted case respect to the full case, as observed in the AFA of the seismicity.

\subsection{Significance of the results}

In order to evaluate the significance of the estimates, we used a surrogate method (Theiler et al., 1986). For each cell we generated one hundred shuffled series of the original sequence, randomly permutating the original interevent time series, thus having the same mean interevent time, but with all the time correlations destroyed; therefore, these surrogate series are characterized by uncorrelated behaviour. We used this procedure to generate surrogate series, in order to maintain identical the probability density function of the interevent times, and, therefore, not to change the first-order properties of the time distribution of the events, whose timecorrelation features we need to test. Randomly shuffling the interevent times practically implies assigning a random value of the time occurrence to the event, but this value is extracted by the same distribution as the original sequence. Our aim is to test whether the calculated estimates of the scaling exponents indicate a significant correlated effect respect to the uncorrelated behaviour displayed by the surrogates; this test is needed especially for those cells with a relatively low number of events, whose correlated behaviour may be due to the limited size of the sample. Being $\alpha(\mathrm{d})$ the scaling exponent of the original sequence, let $\mu_{s}$ and $\sigma_{S}$ indicate the mean and the standard deviation of the scaling exponents calculated for the shuffled sequences. The difference between the exponents before and after the surrogate data test for correlation may be quantified by means of the difference between the original and the mean surrogate value of the scaling exponent, divided by the standard deviation of the surrogate values (Theiler et al., 1986):

$\sigma=\frac{\left|\alpha(d)-\mu_{S}\right|}{\sigma_{S}}$.

$\sigma$ measures how many standard deviations the original exponent is separated from the surrogate data exponent. The larger $\sigma$ the larger the separation between the exponents derived from the surrogate data and the exponent derived from the original data. Thus, larger $\sigma$ value indicates stronger correlation. In order to evaluate the significance of $\sigma$ we also calculated the p-value by means of the formula $\mathrm{p}=\operatorname{erfc}(\sigma / \sqrt{ } 2)$ (Theiler et al., 1986); this is the probability of observing a significance $\sigma$ or larger if the null hypothesis (absence of correlation) is true. This measure furnishes an estimate of the uncertainty of the scaling exponents. Figures 7 (AFA) and 8 (DFA) show the maps of Italy, using the full (a) and depleted (b) catalogues: only the cells with $\mathrm{p}<0.05$ are plotted. We see that almost the same cells, featured by correlated temporal behaviour, characterize both full and depleted catalogues. Furthermore, all the cells, in which the correlation is significant, the correlation is persistent. This suggests the presence of areas in the Italian territory with a strong persistent degree of correlation, which is not only due to the generation of aftershocks, following the occurrence of large shocks, but relies to the underlying time structure of the seismicity of those areas.

\subsection{Discussion of the results}

In the present study, on the basis of a statistical analysis of the scaling properties of the time occurrences of earthquakes, we have identified in the Italian territory areas, which are characterized by a correlated and persistent behavior. The areas, mainly located along the Apennine Chain, are approximately those, which have been characterized in past and recent years 

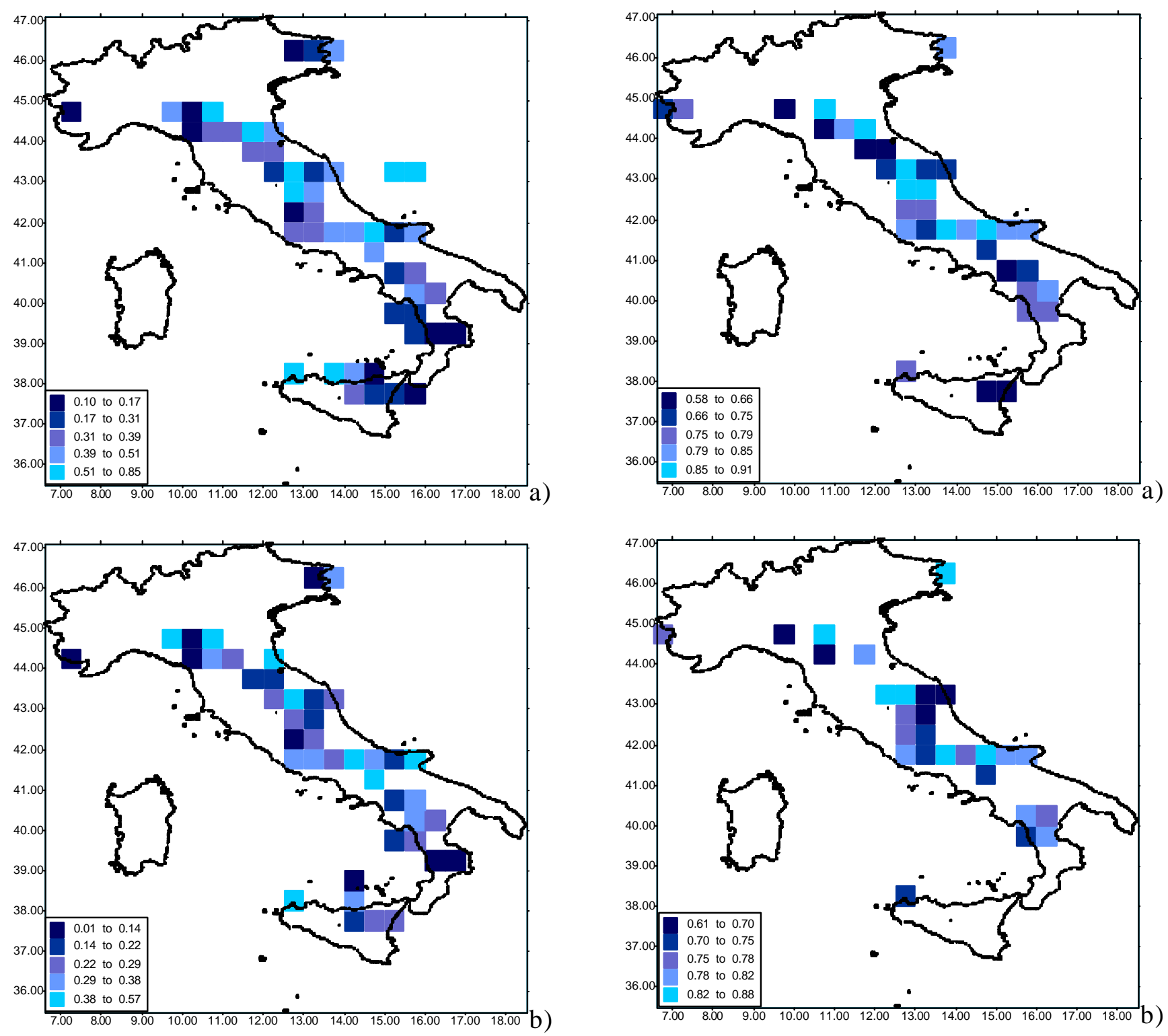

Fig. 7. Map of Italian territory showing the $\alpha$ variability of the cells characterized by $\mathrm{p}<0.05$ : (a) full and (b) aftershock-depleted seismic catalogues.

by strong earthquakes. The most correlated structures are in central Italy, while less correlated behaviour characterizes the seismic variability of southern Italy, except for the north-western part of Sicily (Fig. 7). Figure 8 suggests that persistent feature characterizes all the cells considered, and this means that the seismicity of Italy seems to be "regularly" structured. We observe a higher degree of persistence in central Italy and a lower degree in southern Italy. This classification seems to be in agreement with the recent seismotectonic zonation by Gruppo Nazionale Difesa Terremoti (GNDT) (http://gndt.ingv.it), which discriminates between the zones linked to the inner border of the adriatic-ionic plate in subduction under the apenninic chain (central Italy) and the zones linked to the recent uplift of the apenninic chain

Fig. 8. Map of Italian territory showing the $d$ variability of the cells characterized by $\mathrm{p}<0.05$ : (a) full and (b) aftershock-depleted seismic catalogues.

following a long history of spatio-temporal migration of the foreland system (southern Italy).

The variability of the scaling exponents among the different cells suggest that the time-correlation behaviour is not unique for all the seismic areas, whose time distribution is more or less correlated and more or less persistent.

Bak et al. (2002) have performed a similar analysis on the southern California seismicity, but their aim was the finding of a unified scaling law for earthquakes. In their work, two earthquakes are to be categorized as belonging to a correlated or uncorrelated sequence depending not independently on the waiting time, the magnitude and the length scale, but only on the value of their scaled product. As a consequence, quoting 
Bak et al. (2002), "the short time clustering, commonly referred to as aftershocks, is nothing but the short time limit of the general hierarchical properties of earthquakes".

Our paper furnishes a different perspective of the analysis of the time distribution of seismic events: both the full and the aftershock-depleted seismicity are characterized by almost similar range of values of the scaling exponents, and this indicates that in both cases similar clustering behaviour is detected.

\section{Conclusions}

The seismicity of the Italian territory has been analyzed to reveal the presence and the type of time-correlation phenomena in the data. Two methods have been used, AFA and DFA, which gave consistent results. We used the seismicity data of the 1983-2003 catalogue of the INGV network. We analyzed the full and, in order to avoid bias effects in the estimates, the aftershock-depleted catalogues. The significance of the results has been investigated by means of a surrogate method, that has permitted to discriminate areas with uncorrelated seismicity from those with correlated behaviour. The main findings are summarized as follows: (i) time-scaling behaviour, leading to time-correlated behaviour, is mainly located along the Apennine chain; (ii) the identification of persistence in the time-correlated behaviour of the seismicity implies that the dynamics underlying the seismic phenomenology is characterized by the presence "positive feedback mechanisms"; (iii) significant time correlation is typical of the seismic sequences, not simply related with the mainshock/aftershock mechanisms.

This paper contributes to give another methodological perspective to the seismotectonics of Italy, focusing on the correlation properties of seismicity.

Acknowledgements. The authors are grateful to F. Janssen and an anonymous referee for their valuable criticism and suggestions, which strongly improved the present paper.

Edited by: Ch. Goltz

Reviewed by: F. Janssen and another referee

\section{References}

Bak, P., Christensen, K., Danon, L., and Scanlon, T.: Unified scaling law for earthquakes, Phys. Rev. Lett., 88, 178501, 2002.

Boschi, E., Gasperini, P., and Mulargia, F.: Forecasting where lager crustal earthquakes are likely to occur in Italy in the near future, Bull. Seismol. Soc. Am., 85, 1475-1482, 1995.

Breitenberg, C.: Non-random spectral components in the seismicity of NE Italy, Earth Planet. Sci. Lett., 179, 379-390, 2000.

Cox, D. R. and Isham, V.: Point Processes, Chapman and Hall, London, 1980.

Gardner, J. K. and Knopoff, L.: Statistical search for non-random features of the seismicity of strong earthquakes, Phys. Earth Planet. Int., 12, 291-318, 1976.

Godano, C. and Caruso, V.: Multifractal analysis of earthquake catalogues, Geophys. J. Int., 121, 385-392, 1995.

Gutenberg, B. and Richter, C. F.: Frequency of earthquakes in California: Bull. Seism. Soc. Am., 34, 185-188, 1944.

Havlin, S., Amaral, L. A. N., Ashkenazy, Y., Goldberger, A. L., Ivanov, P. Ch., Peng, C.-K., and Stanley, H. E.: Application of statistical physics to heartbeat diagnosis, Physica A, 274, 99110, 1999.

Kagan, Y. Y. and Jackson, D. D.: Seismic gap hypothesis: ten years after, J. Geophys. Res., 96, 21 419-21 431, 1991.

Lowen, S. B. and Teich, M. C.: Estimation and simulation of fractal stochastic point processes, Fractals, 3, 183-210, 1995.

Öncel, A. O., Main, I. G., Alptekin, Ö, and Cowie, P. A.: Spatial variation in the fractal properties of seismicity in the north Anatolian fault zone, Tectonophysics, 257, 189-202, 1996.

Peng, C.-K., Havlin, S., Stanley, H. E., and Goldberger, A. L.: Quantification of scaling exponents and crossover phenomena in nonstationary heartbeat time series, CHAOS, 5, 82-87, 1995.

Reasenberg, P.: Second-order moment of central California seismicity, 1969-1982, J. Geophys. Res., 90, 5479-5495, 1985.

Telesca, L., Cuomo, V., Lapenna, V., and Macchiato, M.: Identifying space-time clustering properties of the 1983-1997 IrpiniaBasilicata (southern Italy) seismicity, Tectonophysics, 330, 93 102, 2001a.

Telesca, L., Cuomo, V., Lapenna, V., Macchiato, M.: Depthdependent time-clustering behaviour in seismicity of southern California, Geophys. Res. Lett., 28, 4323-4326, 2001 b.

Theiler, J., Eubank, S., Longtin, A., Galdrikian, B., Farmer, J. D.: Testing for nonlinearity in time series: the method of surrogate data, Physica D, 58, 77-94, 1992.

Thurner, S., Lowen, S. B., Feurstein, M. C., Heneghan, C., Feichtinger, H. G., and Teich, M. C.: Analysis, Synthesis, and Estimation of Fractal-Rate Stochastic Point Processes, Fractals, 5, 565-596, 1997.

Utsu, T., Ogata, Y., and Matsu'ura, R. S.: The centenary of the Omori formula for a decay law of aftershock activity, J. Phys. Earth, 43, 1-33, 1995.

Varotsos, P. A., Sarlis, N. V., and Skordas, E. S.: Long-range correlations in the electric signals that precede rupture: Further investigations, Phys. Rev. E, 67, 021109, 2003.

Wilson, T. H. and Dominic, J.: Fractal interrelationships between topography and structure, Earth Surf. Process. Land., 23, 509$525,1998$. 